\title{
Chronic administration of SUMO-1 has negative effects on novel object recognition memory as well as cell proliferation and neuroblast differentiation in the mouse dentate gyrus
}

\author{
DAE YOUNG YOO ${ }^{1 *}$, DAE WON KIM ${ }^{2 *}$, HYUN JUNG KWON ${ }^{2}$, HYO YOUNG JUNG ${ }^{1}$, \\ SUNG MIN NAM ${ }^{1}$, JONG WHI KIM ${ }^{1}$, JIN YOUNG CHUNG ${ }^{3}$, MOO-HO WON $^{4}$, \\ YEO SUNG YOON ${ }^{1}$, SOO YOUNG $\mathrm{CHOI}^{5}$ and IN KOO HWANG ${ }^{1}$
}

\begin{abstract}
${ }^{1}$ Department of Anatomy and Cell Biology, College of Veterinary Medicine, Research Institute for Veterinary Science, Seoul National University, Seoul 08826; ${ }^{2}$ Department of Biochemistry and Molecular Biology, Research Institute of Oral Sciences, College of Dentistry, Kangneung-Wonju National University, Gangneung 25457; ${ }^{3}$ Department of Veterinary Internal Medicine and Geriatrics, College of Veterinary Medicine; ${ }^{4}$ Department of Neurobiology, School of Medicine, Kangwon National University, Chuncheon 24341; ${ }^{5}$ Department of Biomedical Sciences, Research Institute for Bioscience and Biotechnology, Hallym University, Chuncheon 24252, Republic of Korea
\end{abstract}

Received January 28, 2016; Accepted May 9, 2017

DOI: $10.3892 / \mathrm{mmr} .2017 .6946$

\begin{abstract}
Post-translational modifications have been associated with developmental and aging processes, as well as in the pathogenesis of certain diseases. The present study aimed to investigate the effects of small ubiquitin-like modifier 1 (SUMO-1) on hippocampal dependent memory function, cell proliferation and neuroblast differentiation. To facilitate the delivery of SUMO-1 into hippocampal neurons, a transactivator of transcription (Tat)-SUMO-1 fusion protein was constructed and mice were divided into two groups: A vehicle (Tat peptide)-treated group and a Tat-SUMO-1-treated group. The vehicle or Tat-SUMO-1 was administered intraperitoneally to 7-week-old mice once daily for 3 weeks, and a novel object recognition test was conducted following the final treatment; the animals were sacrificed $2 \mathrm{~h}$ following the test for further analysis. Administration of Tat-SUMO-1 significantly decreased exploration of a new object in a novel object recognition test compared with mice in the vehicle-treated group. In addition, cell proliferation and neuroblast differentiation analyses (based on Ki67 and doublecortin
\end{abstract}

Correspondence to: Professor Soo Young Choi, Department of Biomedical Science, Research Institute for Bioscience and Biotechnology, Hallym University, 1 Hallimdaehak-gil, Chuncheon 24252, Republic of Korea

E-mail: sychoi@hallym.ac.kr

Professor In Koo Hwang, Department of Anatomy and Cell Biology, College of Veterinary Medicine, Seoul National University, 1 Kwanak-ro, Kwanak-gu, Seoul 08826, Republic of Korea

E-mail: vetmed2@snu.ac.kr

*Contributed equally

Key words: small ubiquitin-like modifier 1, novel object recognition memory, dentate gyrus, neurogenesis, mice immunohistochemistry, respectively) revealed that the administration of Tat-SUMO-1 significantly reduced cell proliferation and neuroblast differentiation in the dentate gyrus. These results suggested that chronic supplementation of Tat-SUMO-1 affects hippocampal functions by decreasing cell proliferation and neuroblast differentiation in the mouse dentate gyrus.

\section{Introduction}

Post-translational modifications (PTMs) are highly regulated and necessary for complex neuronal processing (1-4). In addition, the regulation of PTMs is one of a number of well-known mechanisms that is required for learning and memory, and for long-term synaptic plasticity (5-8). SUMOylation is one type of PTM, and involves the small ubiquitin-like modifier (SUMO) family, the members of which are ubiquitously expressed in all tissues, including the brain, and has at least three paralogs (SUMO-1, SUMO-2 and SUMO-3) (9). SUMO-1 shares $~ 50 \%$ homology with SUMO-2 and SUMO-3, with some overlap regarding target proteins (10). The pool of unconjugated SUMO-2 and SUMO-3 is generally much larger than that of SUMO-1 in mammalian cells (10).

The hippocampus is considered one of the important regions in the brain that is responsible for spatial memory and navigation $(11,12)$. The hippocampus is of particular interest, as new cells are generated throughout a lifetime and may proliferate and differentiate into neurons in the dentate gyrus (13). SUMOylation is highly regulated through the spatiotemporal expression of SUMO proteins and the SUMO conjugation machinery. The SUMO signaling pathway is involved in important molecular and biological processes, including development and senescence $(14,15)$, and SUMO has been reported to be involved in a number of neuronal pathways, including synaptic formation and transmission, excitability, axonal trafficking and axonal guidance (16-19). 
The brain is a unique organ with its own security system comprising a network of blood vessels and astrocyte end-feet that allow the selective entry of essential nutrients and block other substances (20). However, these unique structures may also limit medications from entering and repairing the injured or diseased brain. Cell-penetrating peptides have been a focus of research in neuroscience due to of their ability to penetrate the blood-brain barrier (BBB) (21-23). Transactivator of transcription (Tat) transduction proteins have been revealed to be able to penetrate the BBB and reach the hippocampus $8 \mathrm{~h}$ following treatment with Tat-fusion proteins in mice (24). In addition, Tat transduction proteins reportedly have no effects on motor coordination, vision or motivation (25).

In the present study, a Tat-SUMO-1 fusion transduction protein was constructed that was able to penetrate the BBB and allowed for the investigation of the effects of SUMO-1 on hippocampal functions, such as novel object recognition, and on cell proliferation and neuroblast differentiation. Immunohistochemical analysis of the proliferation marker protein Ki67 (a marker for active cell cycles, which is absent during $G_{0}$ and early $G_{1}$ ) and the neuronal migration protein doublecortin (DCX, a marker for differentiating neuroblasts) was performed to analyze the expression of these proteins in the dentate gyrus. The results of the present study will allow the role of SUMO-1 in hippocampal neurogenesis in neurological disorders such as stroke and traumatic brain injury to facilitate the rehabilitation of damage in the brain.

\section{Materials and methods}

Experimental animals. Six-week-old male C57BL/6 mice $(n=14)$ were purchased from Japan SLC, Inc. (Shizuoka, Japan). They were housed in a conventional state at room temperature $\left(23^{\circ} \mathrm{C}\right)$ and humidity $(60 \%)$, controlled with a 12-h light/dark cycle. The mice provided with access to food and tap water ad libitum. Handling and caring of the animals conformed to the guidelines established to comply with current international laws and policies (NIH Guide for the Care and Use of Laboratory Animals; National Institutes of Health, Bethesda, MD, USA), and were approved by the Institutional Animal Care and Use Committee of Seoul National University (Seoul, South Korea). All experiments were conducted with an effort to minimize the number of animals used and the suffering caused by the procedures used in the present study.

Tat-SUMO-1 fusion protein expression and purification. The Tat expression vector was prepared as previously described (26). Briefly, the cDNA of human SUMO-1 was amplified by polymerase chain reaction (PCR) from a human liver cDNA library. The sense primer (5'-CTCGAGATG TCTGACCAGGAGGCA-3') contained an XhoI restriction site; the antisense primer (5'-GGATCCCTAAACTGTTGA ATGACCCC-3') contained a BamHI restriction site. The sequences were ligated into the TA-cloning vector (Promega Corporation, Madison, WI, USA), excised, and inserted into the Tat pET-15b (Novagen; Merck KGaA, Darmstadt, Germany) expression vector using T4 DNA ligase (Takara Bio. Inc., Otsu, Japan). The Tat-SUMO-1 plasmid was subsequently cloned into Escherichia coli DH5 $\alpha$ cells (Novagen; Merck $\mathrm{KGaA}$ ) to generate the Tat-SUMO-1 fusion protein (Fig. 1A).

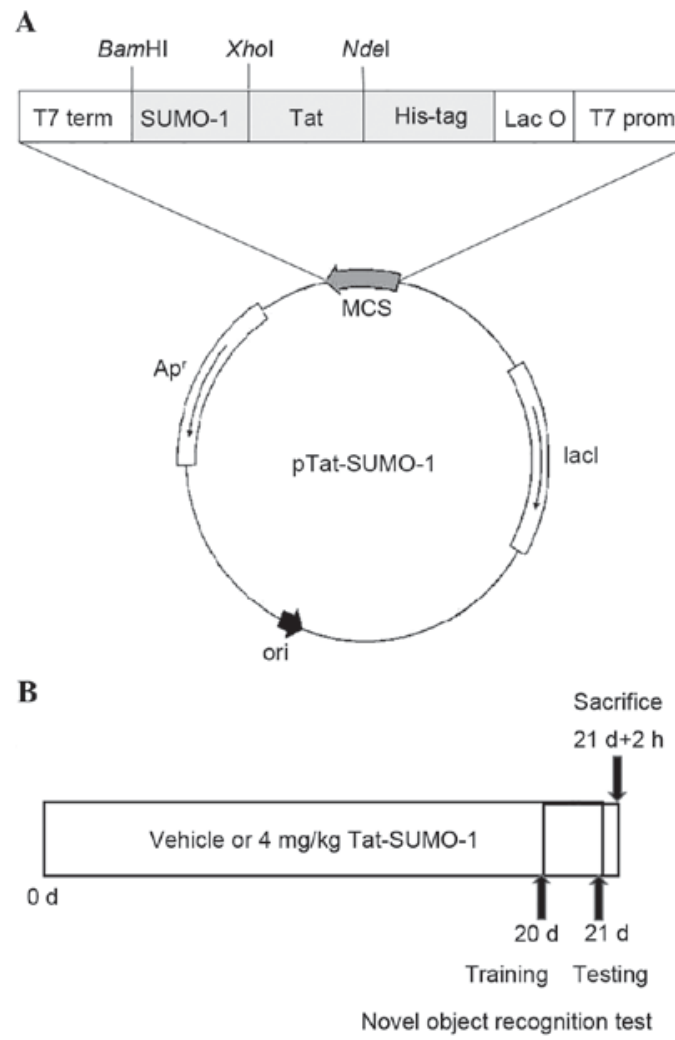

Figure 1. Structure of the Tat-SUMO-1 fusion protein and study-plan schematic. (A) Tat-SUMO-1 fusion protein. The Tat-SUMO-1 expression vector was constructed using the pET-15b vector. (B) Experimental novel object recognition test protocol of the present study. Training trials were performed with two identical objects on day 20, and test trials were performed with one training object and one unfamiliar object on day 21. His, histidine; MCS, multiple cloning site; prom, promoter sequence; SUMO, small ubiquitin-like modifier; Tat, transactivator of transcription; term, terminator sequence.

Control SUMO-1 was generated without the Tat peptide. The Tat peptide was synthesized by Peptron Inc. (Daejeon, South Korea). The recombinant Tat-SUMO-1 plasmid (1 $\mu \mathrm{g})$ was transformed into Escherichia coli BL21 (DE3) competent cells (Novagen; Merck KGaA) and induced with $0.5 \mathrm{mM}$ isopropyl- $\beta$-D-thio-galactoside (Duchefa Biochemie B.V., Haarlem, The Netherlands) at $18^{\circ} \mathrm{C}$ for $>24 \mathrm{~h}$.

Cells were harvested by centrifugation $(6,000 \mathrm{x} \mathrm{g}, 10 \mathrm{~min}$, $\left.4^{\circ} \mathrm{C}\right)$ and sonicated $\left(3\right.$ passes; $\left.4^{\circ} \mathrm{C}\right)$ in lysis buffer $(5 \mathrm{mM}$ imidazole, $500 \mathrm{mM} \mathrm{NaCl}, 20 \mathrm{mM}$ Tris-HCl, pH7.9). The recombinant his-tagged Tat-SUMO-1 fusion protein was purified using a $\mathrm{Ni}^{2+}$-nitrilotriacetic acid Sepharose affinity column (Qiagen, Valencia, CA, USA) according to the manufacturer's protocol. Briefly, clarified cell extracts were loaded at $4^{\circ} \mathrm{C}$. The column was washed with 10 volumes of binding buffer (5 mM imidazole, $500 \mathrm{mM} \mathrm{NaCl}, 20 \mathrm{mM}$ Tris- $\mathrm{HCl}$, $\mathrm{pH}$ 7.9) and 6 volumes of a wash buffer ( $60 \mathrm{mM}$ imidazole, $500 \mathrm{mM} \mathrm{NaCl}, 20 \mathrm{mM}$ Tris-HCl, pH 7.9). The fusion proteins were subsequently eluted using elution buffer $(500 \mathrm{mM}$ imidazole, $500 \mathrm{mM} \mathrm{NaCl}, 20 \mathrm{mM}$ Tris- $\mathrm{HCl}, \mathrm{pH}$ 7.9), followed by desalting with a PD10 column (Amersham, Braunschweig, Germany) according to the manufacturer's protocol. Bovine serum albumin (Sigma-Aldrich; Merck KGaA, Darmstadt, Germany) was used as a standard and protein concentration was measured by Bradford assay (27). 
A Training

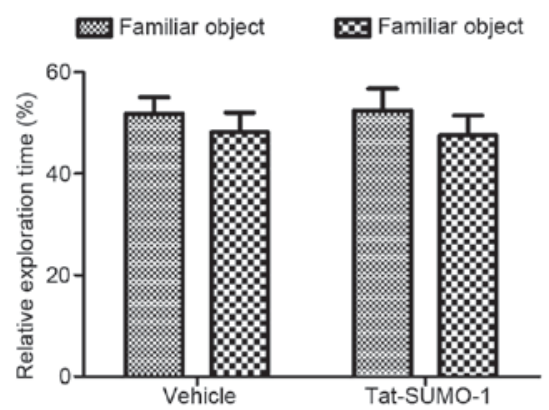

B Testing

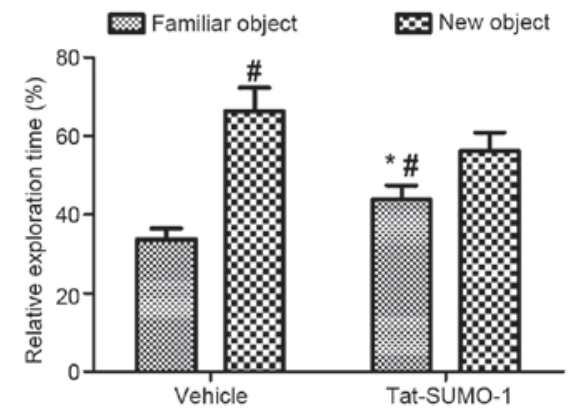

Figure 2. Effects of Tat-SUMO-1 on exploration time of familiar vs. new objects in the novel object recognition test in mice. (A) Vehicle or Tat-SUMO-1 treated mice were initially tested (trained) to identify the effects on exploration time of familiar objects. (B) One of the familiar objects from the training run was replaced with a new object and mouse exploration time was tested again. Data for time of exploration for each object are presented as a percent of total exploration time. All data are shown as a percent of total exploration time \pm standard error of the mean; $\mathrm{n}=7 / \mathrm{group} ;{ }^{*} \mathrm{P}<0.05 \mathrm{vs}$. training test, ${ }^{\sharp} \mathrm{P}<0.05 \mathrm{vs}$. vehicle familiar object. SUMO, small ubiquitin-like modifier; Tat, transactivator of transcription.

Treatment with Tat peptide or Tat-SUMO-1. Seven-week-old mice were divided into 2 groups (n=7/group): i) The vehicle-treatment group, which received a daily intraperitoneal (i.p.) injection of glycerol with Tat peptide $(4 \mathrm{mg} / \mathrm{kg}$ ) for 3 weeks; and ii) the Tat-SUMO-1-treatment group, which received a daily i.p. injection of Tat-SUMO-1 (4 mg/kg) for 3 weeks. This schedule was adopted as DCX is expressed exclusively in the immature neurons between 1 and 28 days of cell age $(28,29)$.

Novel object recognition test. One hour following vehicle or Tat-SUMO-1 treatment on day 20 (Fig. 1B), the training trial was performed. Mice ( $n=7 /$ group) were placed in a $45 \times 45 \times 30 \mathrm{~cm}$ acrylic box with three opaque walls and one transparent wall, and allowed to explore two identical objects for $5 \mathrm{~min}$ each. This training process was repeated once. A total of $24 \mathrm{~h}$ following the training open-field trial, the test trial was performed; one of the two familiar objects was replaced with a new one, and the mice were allowed to explore them for $5 \mathrm{~min}$. Relative exploration time was calculated as follows: Relative exploration time in first and second day trials=[time observed each object (familiar or new)/time observing both objects]x 100. The animals were sacrificed $2 \mathrm{~h}$ following completion of the novel object recognition test.

Tissue processing. For histological analysis, mice from the vehicle and Tat-SUMO-1-treated groups ( $n=7 /$ group) were anesthetized with urethane ( $2 \mathrm{mg} / \mathrm{kg}$; Sigma-Aldrich; Merck $\mathrm{KGaA}$ ) at $2 \mathrm{~h}$ following the novel object recognition test and perfused transcardially with PBS (0.1 M; pH 7.4), followed by $4 \%$ paraformaldehyde in phosphate buffer $(0.1 \mathrm{M}$; $\mathrm{pH} 7.4)$. Brains were removed and post-fixed in $4 \%$ paraformaldehyde in phosphate buffer for $12 \mathrm{~h}$ at $4^{\circ} \mathrm{C}$. Brain tissues were cryoprotected by infiltration with $30 \%$ sucrose overnight at $4^{\circ} \mathrm{C}$. Brain sections of $(30 \mu \mathrm{m})$ were serially cut in the coronal plane using a cryostat (Leica Microsystems GmbH, Wetzlar, Germany). Sections were collected in six-well plates containing PBS until further processing.

Immunohistochemistry. To obtain accurate data for immunohistochemistry, free-floating brain sections were carefully processed under the same conditions. For each animal, tissue sections were selected between -1.46 and $-2.46 \mathrm{~mm}$ posterior to bregma by referring to the mouse atlas by Franklin and Paxinos (30). A total of 10 sections, $90 \mu \mathrm{m}$ apart from each other, were sequentially treated with $0.3 \%$ hydrogen peroxide in PBS for $30 \mathrm{~min}$ at $25^{\circ} \mathrm{C}$ and $10 \%$ normal goat or rabbit serum (Vector Laboratories, Inc., Burlingame, CA, USA) in $0.05 \mathrm{M}$ PBS for $1 \mathrm{~h}$ at $25^{\circ} \mathrm{C}$. Sections were incubated overnight with rabbit anti-Ki67 antibody (1:1,000; ab15580, Abcam, Cambridge, UK) or goat anti-DCX antibody (1:50; sc-8066, Santa Cruz Biotechnology, Inc., Dallas, TX, USA) at $4^{\circ} \mathrm{C}$, and subsequently exposed to biotinylated rabbit anti-goat or goat anti-rabbit immunoglobulin $\mathrm{G}$ at $25^{\circ} \mathrm{C}$ for $2 \mathrm{~h}$ (1:200; BA-5000 or BA-1000, Vector Laboratories, Inc.) and streptavidin peroxidase complex (1:200, Vector Laboratories, Inc.) at $25^{\circ} \mathrm{C}$ for $2 \mathrm{~h}$. The sections were visualized by reaction with 3,3'-diaminobenzidine tetrahydrochloride (Sigma-Aldrich; Merck KGaA).

Ki67- and DCX-positive cell counts were performed in the hippocampal subgranular zone for each section of the dentate gyrus using a computer-based CCD camera and Optimas v 6.5 image analysis software (CyberMetrics, Phoenix, AZ, USA). Cell counts from all sections from each of the mice were averaged.

Statistical analysis. The data are presented as the mean \pm standard error of the mean. Differences among the means were statistically analyzed by two-way analysis of variance followed by Bonferroni's post-hoc test to elucidate the effects of Tat-SUMO-1 on novel object recognition. A paired Student's t-test was employed to observe alterations in cell proliferation and neuroblast differentiation in mice. All statistical tests were performed using GraphPad Prism software v 5.01 (GraphPad Software, Inc., La Jolla, CA, USA). P<0.05 was considered to indicate a statistically significant difference.

\section{Results}

Effects of Tat-SUMO-1 on object recognition memory. During the training period, mice from the vehicle and Tat-SUMO-1-treated groups spent similar amounts of time (18.74 vs. $19.01 \mathrm{sec}$, respectively; Fig. 2) exploring two identical objects $(\mathrm{F}=0.03, \mathrm{P}=0.8734)$. During the test period, mice in both the vehicle- and the Tat-SUMO-1-treated groups spent 


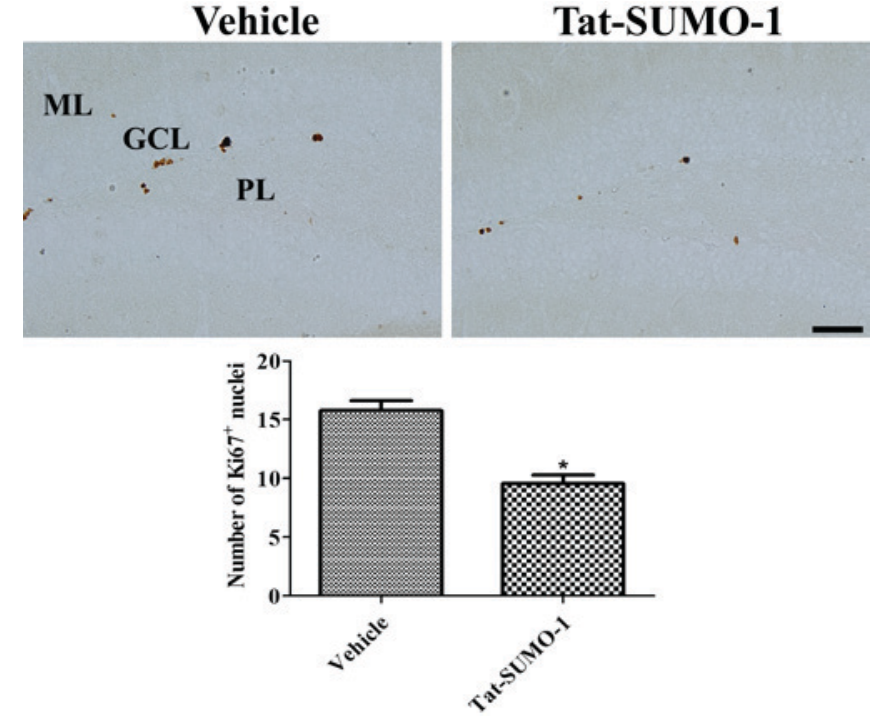

Figure 3. Immunohistochemical analysis of Ki67 in the dentate gyrus. Ki67 immunoreactive-positive nuclei in vehicle- and Tat-SUMO-1-treatment groups are mainly detected in the subgranular zone of dentate gyrus. Scale bar, $50 \mu \mathrm{m}$ In Tat-SUMO-1 mice, the average number of Ki67-positive nuclei per section are fewer in number as compared with the vehicle-treated group. Data are presented as the mean \pm standard error of the mean; $n=7 /$ group; ${ }^{*} \mathrm{P}<0.05$ vs. Vehicle group). GCL, granule cell layer; ML, molecular layer; PL, polymorphic layer; SUMO, small ubiquitin-like modifier; Tat, transactivator of transcription.

significantly more time exploring the new object than the familiar one, compared with time spent with the objects in the training period $(\mathrm{F}=5.20, \mathrm{P}=0.03128)$. The relative exploration time in the Tat-SUMO-1-treated group was less than that for the vehicle-treated group and statistical significance was identified between vehicle and Tat-SUMO-1-treated groups $(\mathrm{P}<0.05)$.

Effects of Tat-SUMO-1 on cell proliferation. Ki67 immunoreactive-positive nuclei were observed in the subgranular zone of the dentate gyrus. In the Tat-SUMO-1-treated group, significantly fewer Ki67-positive nuclei were detected compared with the vehicle-treated group (9.6 vs. 15.8, respectively; Fig. 3).

Effects of Tat-SUMO-1 on neuroblast differentiation. DCX immunoreactive-positive neuroblasts were detected in the dentate gyrus. Many of the DCX-positive neuroblasts had cytoplasm in the subgranular zone with dendrites extended across the granule cell layer into molecular layer of the dentate gyrus. In the Tat-SUMO-1-treated group, DCX-positive neuroblasts were less abundant and had poorly developed dendrites in the dentate gyrus. The average number of DCX immunoreactive neuroblasts was significantly decreased in the Tat-SUMO-1-treated group compared with the vehicle-treated group (89.5 vs. 145.0 per section, respectively; Fig. 4).

\section{Discussion}

Modulation of PTMs is a crucial process for homeostasis, development and metabolism in several organs, including the brain. Several previous studies have suggested that SUMOylation is one of the key factors in synaptic plasticity

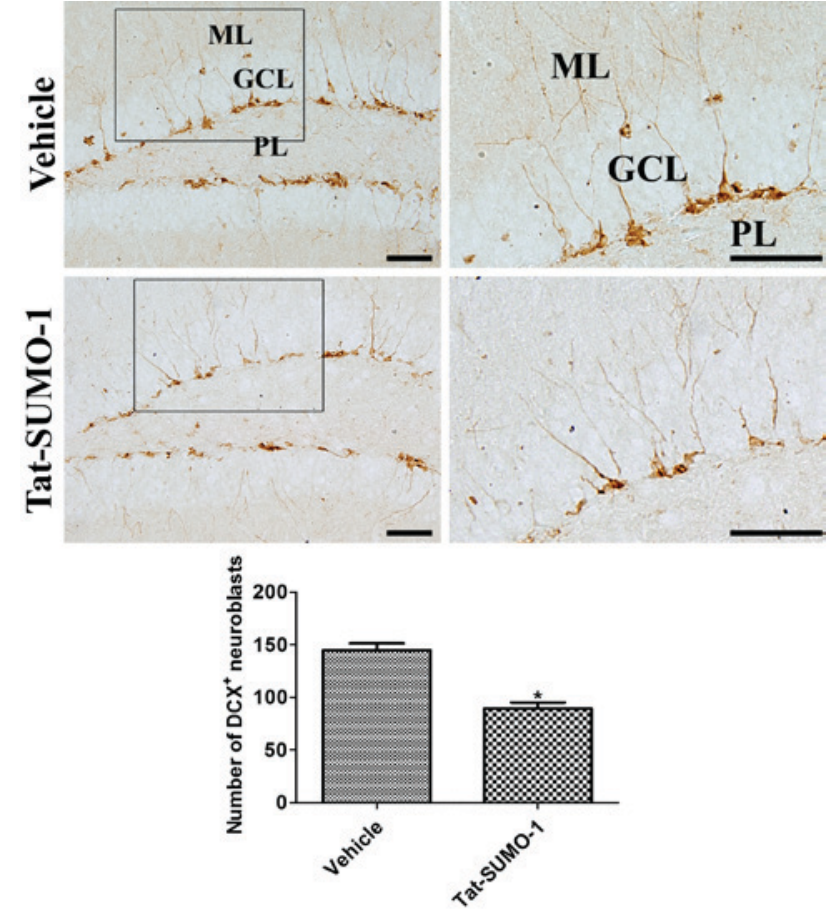

Figure 4. Immunohistochemistry for DCX in the dentate gyrus. DCX immunoreactive-positive neuroblasts are abundantly observed in the dentate gyrus. Fewer DCX-positive neuroblasts and their dendrites are observed in the dentate gyrus of the Tat-SUMO-1-treated group as compared with those in the vehicle-treated group. Scale bar $=50 \mu \mathrm{m}$. Number of DCX-positive neuroblasts per section for each group. Data are presented as the mean \pm standard error of the mean; $n=7 /$ group; ${ }^{*} \mathrm{P}<0.05$ vs. Vehicle. DCX, doublecortin; GCL, granule cell layer; ML, molecular layer; PL, polymorphic layer; SUMO, small ubiquitin-like modifier; Tat, transactivator of transcription.

in the brain $(31,32)$. SUMOylation has been demonstrated to induce important changes in Alzheimer's disease-associated proteins, such as tau and amyloid- $\beta$ precursor protein $(33,34)$. SUMO-1 mRNA levels, as well as SUMO-1-ylation, are increased in the hippocampus of 6-month-old $\operatorname{Tg} 2576$ Alzheimer's disease model mice as compared with wild-type mice (35). In amyloid- $\beta$ precursor protein transgenic (Tg6799) mice, free SUMO-1 protein levels are significantly increased in the cortical tissue at 18 months old (36). In subjects with dementia, SUMO-1 levels were significantly increased in the serum as compared with the levels of SUMO-1 in similarly aged healthy controls (37).

In the present study, the effects of Tat-SUMO-1 on hippocampal function were analyzed using the novel object recognition paradigm, as the test depends on the integrity of the hippocampus (38) and that newly generated adult-born neurons of the dentate gyrus contribute to hippocampus-dependent memory (39). Chronic administration of Tat-SUMO-1 significantly decreased novel object recognition memory. In SUMO-1 transgenic mice, genetic overexpression of SUMO-1 did not result in alterations to the behavioral phenotype or open field responses, except for fear conditioning (40). By contrast, the inhibition of SUMOylation by infusions of dominant negative Tat-Ubc9 significantly impaired hippocampus-dependent reference memory, based on the Morris water-maze test (25). In addition, knockdown of SUMO led to impairment in episodic memory processes, contextual and cued fear conditioning, and fear-potentiated 
startle (41). These conflicting results suggest that the overexpression or lack of SUMO may affect memory processing and have chronic deleterious effects.

SUMOylation has been implicated in neurodevelopmental and neurodegenerative processes in the brain (16-19). In a recent study, SUMO-1 transgenic mice exhibited impairment in both short-term synaptic plasticity, based on paired pulse facilitation measurements, and basal synaptic transmission, based on the input-output relationship plots (40). In addition, synaptic density in pyramidal cells was reduced by $70 \%$ in the SUMO-1 transgenic mice based on Golgi staining as compared with that in the wild-type mice (40). In the present study, the effects of Tat-SUMO-1 on cell proliferation and neuroblast differentiation in the dentate gyrus were observed. Administration of Tat-SUMO-1 significantly reduced the number of proliferating cells and differentiated neuroblasts in the dentate gyrus, and significantly decreased the time spent exploring a new object in the novel object recognition test. This study suggests that SUMO-1 is closely involved in hippocampal neurogenesis and functioning; controlling neurogenesis by modulation of SUMOylation may be new strategy to overcome neurological disorders.

\section{Acknowledgements}

The present study was supported by National Research Foundation of Korea (NRF) grant, funded by the Korean government Ministry of Science, ICT \& Future Planning (MSIP) (grant no. NRF-2016R1A2B4009156). This research was also supported by a Priority Research Centers Program grant from the NRF of Korea (grant no. NRF-2009-00,93812), as funded by MSIP in the Republic of Korea.

\section{References}

1. Gwizdek C, Cassé F and Martin S: Protein sumoylation in brain development, neuronal morphology and spinogenesis. Neuromolecular Med 15: 677-691, 2013.

2. Luo J, Ashikaga E, Rubin PP, Heimann MJ, Hildick KL, Bishop P, Girach F, Josa-Prado F, Tang LT, Carmichael RE, et al: Receptor trafficking and the regulation of synaptic plasticity by SUMO. Neuromolecular Med 15: 692-706, 2013.

3. Cho Y and Cavalli V: HDAC signaling in neuronal development and axon regeneration. Curr Opin Neurobiol 27: 118-126, 2014.

4. Santos AI, Martínez-Ruiz AI and Araújo IM: S-nitrosation and neuronal plasticity. Br J Pharmacol 172: 1468-1478, 2015.

5. Soderling TR and Derkach VA: Postsynaptic protein phosphorylation and LTP. Trends Neurosci 23: 75-80, 2000.

6. DiAntonio A and Hicke L: Ubiquitin-dependent regulation of the synapse. Annu Rev Neurosci 27: 223-246, 2004.

7. Fukata $\mathrm{Y}$ and Fukata M: Protein palmitoylation in neuronal development and synaptic plasticity. Nat Rev Neurosci 11: 161-175, 2010.

8. Routtenberg A and Rekart JL: Post-translational protein modification as the substrate for long-lasting memory. Trends Neurosci 28: 12-19, 2005.

9. Droescher M, Chaugule VK and Pichler A: SUMO rules: Regulatory concepts and their implication in neurologic functions. Neuromolecular Med 15: 639-660, 2013.

10. Saitoh H and Hinchey J: Functional heterogeneity of small ubiquitin-related protein modifiers SUMO-1 versus SUMO-2/3. J Biol Chem 275: 6252-6258, 2000.

11. Moser MB and Moser EI: Functional differentiation in the hippocampus. Hippocampus 8: 608-619, 1998.

12. Schmajuk NA: Role of the hippocampus in temporal and spatial navigation: An adaptive neural network. Behav Brain Res 39: 205-229, 1990.
13. Gage FH, Kempermann G, Palmer TD, Peterson DA and Ray J: Multipotent progenitor cells in the adult dentate gyrus. J Neurobiol 36: 249-266, 1998.

14. Andreou AM and Tavernarakis N: Roles for SUMO modification during senescence. Adv Exp Med Biol 694: 160-171, 2010.

15. Lomelí H and Vázquez M: Emerging roles of the SUMO pathway in development. Cell Mol Life Sci 68: 4045-4064, 2011.

16. Martin S, Wilkinson KA, Nishimune A and Henley JM: Emerging extranuclear roles of protein SUMOylation in neuronal function and dysfunction. Nat Rev Neurosci 8: 948-959, 2007.

17. Craig TJ and Henley JM: Protein SUMOylation in spine structure and function. Curr Opin Neurobiol 22: 480-487, 2012.

18. Wilkinson KA, Nakamura Y and Henley JM: Targets and consequences of protein SUMOylation in neurons. Brain Res Rev 64: 195-212, 2010.

19. Plant LD, Dowdell EJ, Dementieva IS, Marks JD and Goldstein SA: SUMO modification of cell surface Kv2.1 potassium channels regulates the activity of rat hippocampal neurons. J Gen Physiol 137: 441-454, 2011.

20. Ballabh P, Braun A and Nedergaard M: The blood-brain barrier: An overview: Structure, regulation and clinical implications. Neurobiol Dis 16: 1-13, 2004.

21. Kilic E, Kilic U and Hermann DM: TAT fusion proteins against ischemic stroke: Current status and future perspectives. Front Biosci 11: 1716-1721, 2006.

22. Fonseca SB, Pereira MP and Kelley SO: Recent advances in the use of cell-penetrating peptides for medical and biological applications. Adv Drug Deliv Rev 61: 953-964, 2009.

23. Stalmans S, Bracke N, Wynendaele E, Gevaert B, Peremans K, Burvenich C, Polis I and De Spiegeleer B: Cell-penetrating peptides selectively cross the blood-brain barrier in vivo. PLoS One 10: e0139652, 2015.

24. Eum WS, Kim DW, Hwang IK, Yoo KY, Kang TC, Jang SH, Choi HS, Choi SH, Kim YH, Kim SY, et al: In vivo protein transduction: Biologically active intact pep-1-superoxide dismutase fusion protein efficiently protects against ischemic insult. Free Radic Biol Med 37: 1656-1669, 2004.

25. Lee L, Dale E, Staniszewski A, Zhang H, Saeed F, Sakurai M, $\mathrm{Fa}^{\prime} \mathrm{M}$, Orozco I, Michelassi F, Akpan N, et al: Regulation of synaptic plasticity and cognition by SUMO in normal physiology and Alzheimer's disease. Sci Rep 4: 7190, 2014.

26. Kwon HY, Eum WS, Jang HW, Kang JH, Ryu J, Ryong Lee B, Jin LH, Park J and Choi SY: Transduction of Cu, Zn-superoxide dismutase mediated by an HIV-1 Tat protein basic domain into mammalian cells. FEBS Lett 485: 163-167, 2000.

27. Bradford MM: A rapid and sensitive method for the quantification of microgram quantities of protein utilizing the principle of protein-dye binding. Anal Biochem 72: 248-254, 1976.

28. Brown JP, Couillard-Després S, Cooper-Kuhn CM, Winkler J, Aigner L and Kuhn HG: Transient expression of doublecortin during adult neurogenesis. J Comp Neurol 467: 1-10, 2003.

29. Couillard-Despres S, Winner B, Schaubeck S, Aigner R, Vroemen M, Weidner N, Bogdahn U, Winkler J, Kuhn HG and Aigner L: Doublecortin expression levels in adult brain reflect neurogenesis. Eur J Neurosci 21: 1-14, 2005.

30. Franklin KBJ and Paxinos G (eds): The Mouse Brain In Stereotaxic Coordinates. 3rd edition. San Diego, Academic Press, 1997

31. Chamberlain SE, González-González IM, Wilkinson KA, Konopacki FA, Kantamneni S, Henley JM and Mellor JR: SUMOylation and phosphorylation of GluK2 regulate kainate receptor trafficking and synaptic plasticity. Nat Neurosci 15: 845-852, 2012.

32. Martin S, Nishimune A, Mellor JR and Henley JM: SUMOylation regulates kainate-receptor-mediated synaptic transmission. Nature 447: 321-325, 2007.

33. Georgopoulou N, McLaughlin M, McFarlane I and Breen KC: The role of post-translational modification in beta-amyloid precursor protein processing. Biochem Soc Symp: 23-36, 2001.

34. Marcus JN and Schachter J: Targeting post-translational modifications on tau as a therapeutic strategy for Alzheimer's disease. J Neurogenet 25: 127-133, 2011.

35. Nisticò R, Ferraina C, Marconi V, Blandini F, Negri L, Egebjerg J and Feligioni M: Age-related changes of protein SUMOylation balance in the A $\beta$ PP Tg2576 mouse model of Alzheimer's disease. Front Pharmacol 5: 63, 2014. 
36. Yun SM, Cho SJ, Song JC, Song SY, Jo SA, Jo C, Yoon K, Tanzi RE, Choi EJ and Koh YH: SUMO1 modulates A $\beta$ generation via BACE1 accumulation. Neurobiol Aging 34: 650-662, 2013.

37. Cho SJ, Yun SM, Lee DH, Jo C, Ho Park M, Han C and Ho Koh Y: Plasma SUMO1 protein is elevated in Alzheimer's disease. J Alzheimers Dis 47: 639-643, 2015.

38. Squire LR, Wixted JT and Clark RE: Recognition memory and the medial temporal lobe: A new perspective. Nat Rev Neurosci 8: $872-883,2007$.
39. Leuner B and Gould E: Structural plasticity and hippocampal function. Annu Rev Psychol 61: 111-140, 2010.

40. Matsuzaki S, Lee L, Knock E, Srikumar T, Sakurai M, Hazrati LN, Katayama T, Staniszewski A, Raught B, Arancio O and Fraser PE: SUMO1 affects synaptic function, spine density and memory. Sci Rep 5: 10730, 2015.

41. Wang L, Rodriguiz RM, Wetsel WC, Sheng H, Zhao S, Liu X, Paschen W and Yang W: Neuron-specific Sumol-3 knockdown in mice impairs episodic and fear memories. J Psychiatry Neurosci 39: 259-266, 2014. 